Revista Calidad en la Educación Superior Programa de Autoevaluación Académica Universidad Estatal a Distancia, Costa Rica ISSN 1659-4703

revistacalidad@uned.ac.cr

\title{
UNIÓN INTERUNIVERSITARIA PARA EL FORTALECIMIENTO DE LAS OLIMPIADAS COSTARRICENSES DE CIENCIAS BIOLÓGICAS COMO PROCESO DE FORMACIÓN DE LOS FUTUROS PROFESIONALES EN EL ÁREA DE LAS CIENCIAS Y LA ACTUALIZACIÓN DE LOS CONOCIMIENTOS DE LOS Y LAS DOCENTES DE SECUNDARIA SOBRE BIOLOGÍA
}

INTERUNIVERSITY UNION FOR STRENGTHENING THE COSTA RICAN
OLYMPICS OF BIOLOGICAL SCIENCES AS A FORMATION PROCESS FOR
FUTURE PROFESSIONALS IN THE AREA OF SCIENCES AND
PROFESSIONAL DEVELOPMENT FOR HIGHSCHOOL TEACHERS IN BIOLOGY

\author{
Magaly Rodríguez Calvo ${ }^{1}$ marodriguez@uned.ac.cr \\ Universidad Estatal a Distancia, Costa Rica \\ José M. Pereira Chaves ${ }^{2}$ jose.pereira.chaves@una.cr \\ Universidad Nacional, Costa Rica
}

\author{
Volumen 6, número 1 \\ Mayo 2015 \\ Pp. $91-118$
}

Recibido: 20 de mayo, 2014

Aprobado: 25 de marzo, 2015

${ }^{1}$ Magaly Rodríguez Calvo, Máster en Administración Educativa y Licenciada en Biología Tropical

2José M. Pereira Chaves, Doctor en Educación, Máster en Ciencias Marinas y Licenciado en Enseñanza de las Ciencias 
Unión interuniversitaria para el fortalecimiento de las Olimpiadas Costarricenses de Ciencias Biológicas como proceso de formación de los futuros profesionales en el área de las ciencias y la actualización de los conocimientos de los y las docentes de secundaria sobre biología

Magaly Rodríguez Calvo y José M. Pereira Chaves

\title{
Resumen
}

Las Olimpiadas Costarricenses de Ciencias Biológicas promueven el estudio activo, participativo y significativo de las ciencias biológicas a nivel de la enseñanza media y preuniversitaria y potencian el nivel cognitivo mediante competencias académicas dirigidas a estudiantes y docentes de secundaria, con el objetivo de mejorarla calidad educativa y fortalecer sus conocimientos y la adquisición de nuevas destrezas y habilidades teórico-prácticas relacionadas con esta ciencia.

Es una actividad que genera espacios de participación en todos los centros educativos, tanto públicos como privados, de las veintiún Sedes Regionales del país. Esta competencia se divide en dos categorías: la A, para estudiantes de los nivelesde décimo, undécimo y duodécimo; y la $B$, para estudiantes de los niveles de octavo, noveno y décimo.

La unión interuniversitaria entre la Universidad de Costa Rica, la Universidad Estatal a Distancia y la Universidad Nacional ha logrado consolidar y fortalecer las Olimpiadas Costarricenses de Ciencias Biológicas, proyectándolas como un evento de gran alcance y significado para el desarrollo de las ciencias biológicas en la educación y en la sociedad costarricenses, desarrollando diversas gestiones para estudiantes de secundaria y para docentes de educación media. Esta actividad tiene gran valor en la labor extensiva de las universidades.

El establecimientode un equipo de colaboradores y colaboradoras entre las universidades involucradas en esta actividad de extensión, así como deotras instituciones gubernamentales y no gubernamentales relacionadas con la educación, ha fortalecido las Olimpiadas y ha logrado aumentar la participación año a año de estudiantes y docentes de secundaria.

Palabras claves: estudiantes, docentes, secundaria, universidades, enseñanzaaprendizaje, educación, capacitaciones, biología, ciencias

\begin{abstract}
The Biological Sciences Olympics of Costa Rica promote active, participatory and significant study of the biological sciences at the high school and pre-university education level, encouraging the cognitive level through academic competitions for students and teachers, with the aim of improving, both the quality of education and strengthening their knowledge and acquire new and theoretical and practical skills related to this Science.

This activity generates opportunities for the participation of all public and private schools, at the 21 regional offices around the country. This competition is divided into two categories, A for students of X, XI and XII level and B for students of VIII, IX, and X level.
\end{abstract}


The coalition between University of Costa Rica, State Distance University and National University, has managed to consolidate and strengthen the Biological Sciences Olympics of Costa Rica, projecting it as an event of great scope and significance for the development of the biological sciences in the education and in the society of the country, developing various approaches for high school students and middle school teachers, being this activity of great value in the extensive work of the universities

By setting up a team of collaborators from the involved universities and other governmental and non-governmental institutions related to education in this extension activity, has strengthened the Olympiad per se, and achieved increased participation of the students and middle school teachers.

Keywords: Students, teachers, high school, university, teaching-learning, education, training, biology, science

Desde hace muchos años las competencias académicas a nivel científico son de extensa trayectoria y relevancia mundial. En América Latina, países como México, Argentina y Brasil tienen la más amplia experiencia con sus excelentes niveles organizativos, tanto para sus fases nacionales como internacionales.

Es por ello que, debido a que se cuenta con participaciones a nivel latinoamericano en competencias científicas y con la experiencia de la Universidad Nacional (UNA) en la organizaciónde justas de las Olimpiadas de Matemática, Física y Química a nivel nacional —todas ellas con proyección internacional, ya sea a nivel centroamericano, Iberoamericano o mundial-, se toma la iniciativa en el 2007 de organizar la primera competencia académica en el área de la Biología, una propuesta de trabajo final de graduación de dos estudiantes, Esteban Espinoza, del grado de licenciatura, y Ana Lilliam Zepeda, del grado de bachillerato. Dicha propuesta se denomina I Olimpiada Costarricense de Biología, y surge con apoyo de la Facultad de Ciencias Exactas y Naturales, la Vicerrectoría 
de Extensión y la Escuela de Ciencias Biológicas de la Universidad Nacional (Escuela de Ciencias Biológicas, 2008).

Sin duda era imprescindible impulsar en Costa Rica las competencias en el área de las ciencias, ya que precisamente la materia de Biología la escoge alrededor de un $80 \%$ de la población estudiantil para realizar el examen de bachillerato. De este modo, se pretendía que el proyecto abarcara esas áreas inconclusas en el desarrollo de la Biología en secundaria, brindando un espacio de actualización, competición y superación académica.

Por tanto, las Olimpiadas Costarricenses de Ciencias Biológicas (OLICOCIBI) constituyen una competencia intelectual dirigida a jóvenes estudiantes del III Ciclo de la Educación General Básica y del Ciclo Diversificado, estableciendo dos categorías: la $A$, para estudiantes de décimo, undécimo y duodécimo; y la categoría B, para alumnos y alumnas de octavo, noveno y décimo. Las OLICOCIBI tienen como propósitos primordial es la estimulación del estudio de las ciencias biológicas y el descubrimiento de jóvenes con talento en la disciplina (Escuela de Ciencias Biológicas, 2008).

Aunque fue una actividad que nació en la Universidad Nacional (UNA), esta institución logró la vinculación de otras instituciones relacionadas con la educación, como el Ministerio de Ciencia y Tecnología (MICIT), el Ministerio de Educación Pública (MEP), el Consejo Nacional de Ciencia y Tecnología (CONICIT), la Universidad Estatal a Distancia (UNED) y la Universidad de Costa Rica (UCR).Para incorporarlas en este proyecto de extensión se consolidó el desarrollo de la competencia a nivel nacional con una regularidad anual, estimulando la participación de docentes y estudiantes en cuanto a la posibilidad de capacitarse y competir, tanto a nivel nacional como internacional. 
Este tipo de competencias como las Olimpiadas de Biología son de gran importancia a nivel de la enseñanza media y preuniversitaria, ya que enriquecen el área de las ciencias, consiguiendo motivar de manera activa, participativa y significativaa estudiantes y docentes, logrando que se dé un intercambio de ideas, y así complementar los conocimientos teóricos con los prácticos para fortalecer el potencial cognitivo en la educación secundaria.

De ahí que algunos de los propósitos formulados desde el inicio del proyecto se han podido alcanzar exitosamente, y más aún con la participación interuniversitaria (otras instituciones que han colaborado en algunos procesos específicos), consiguiendo que las OLICOCIBI se destaquen en las siguientes intensiones:

- Estimular el estudio de la Biología a nivel nacional

- Promover el desarrollo vocacional de estudiantes talentosos en la Biología para su incorporación en las diferentes carreras que ofrecen las universidades en el área de las ciencias.

- Fomentar el intercambio de experiencias entre docentes de la Enseñanza de la Biología como una práctica para retroalimentar sus técnicas metodológicas de enseñanza-aprendizaje

- Incentivar en las personas participantes una actitud responsable hacia los avances científicos en el campo de la biología

- Impulsar la participación de nuestro país en eventos olímpicos a nivel internacional (Escuela de Ciencias Biológicas, 2008).

Se logra visualizar que la articulación interuniversitaria ha conseguido fortalecer el proyecto de las Olimpiadas de Biología para que los participantes interesados de los diferentes colegios logren compartir experiencias similares en las ciencias y aumenten su vocación hacia el fortalecimiento de la educación universitaria 
científico-tecnológica. Por ello, las OLICOCIBI ayudan a los y las estudiantes como valor agregado a la escogencia en su carrera profesional, siendo este aspecto provechoso para el desarrollo de futuros profesionales en el área de las ciencias.

Dentro de las propuestas a ejecutar en las Olimpiadas de Biología, como entidad de Educación Superior, está la de establecer nuevos vínculos de cooperación con el Sistema de Educación Secundaria. Esto conlleva a la realizaciónde programas de capacitación y talleres de actualización para el personal docente, tanto de colegios urbanos como rurales, que imparte las materias relacionados con la Biología, para mejorar el proceso de enseñanza y aprendizaje en la disciplina científica y, por ende, un sobresaliente nivel pedagógico.

\section{Unión de universidades versus mayor participación de estudiantes de colegios de secundaria en las Olimpiadas de Biología}

Las OLICOCIBI desde el año 2008 organizan una competencia intelectual dirigida a jóvenes estudiantes del III Ciclo de la Educación General Básica y del Ciclo Diversificado, en la que se estimula el estudio de las ciencias biológicas para descubrir jóvenes con talento en esta área.

Asimismo, constituye un marco oportuno para el intercambio de experiencias entre el profesorado y el estudiantado. Además favorece el despertar de talentos científicos de futuros y futuras profesionales en el país (Pereira, Camacho y Muñoz, 2013).

El desarrollode este proyecto brinda la oportunidad de establecer nuevos vínculos de cooperación entre entidades de Educación Superior y el Sistema de Educación Secundaria. 
Al crear estos vínculos se alcanzó en el 2010 la articulación interuniversitaria, ya que se unieron al proyecto de extensión originado en la UNA dos de las universidades estatales:la Universidad de Costa Rica (UCR) y la Universidad Estatal a Distancia (UNED); con el apoyo de estas instituciones se ha logrado ampliar la divulgación a más colegios que pertenecen a las veintiún regionales que tiene establecidas el Ministerio de Educación Pública (MEP). Gracias a esto se ha conseguido entregar más cantidad de material informativo, con lo cual se obtiene mayor visitación a los colegios yaumentala participación de estos en las OLICOCIBI.

En las figuras 1 y 2 se puede visualizar la cantidad de estudiantes que se han inscrito por año, así como la cantidad de colegios participantes.Se observa que a través de los años se ha incrementado la cantidad de estudiantesy de colegios participantes, destacando que a partir de 2010 aumentó considerablemente la participación debido a que se unieron las universidades mencionadas con anterioridad. De ahí que se determine que al brindar más divulgación de las Olimpiadas se propicia un mayor interés en estudiantes y colegios por participar en la competencia nacional, adquiriendo las universidades una buena organización y adecuada cobertura de publicidad a nivel nacional. 
Unión interuniversitaria para el fortalecimiento de las Olimpiadas Costarricenses de Ciencias Biológicas como proceso de formación de los futuros profesionales en el área de las ciencias y la actualización de los conocimientos de los y las docentes de secundaria sobre biología

Magaly Rodríguez Calvo y José M. Pereira Chaves

Figura 1. Cantidad de estudiantes que han participado por año

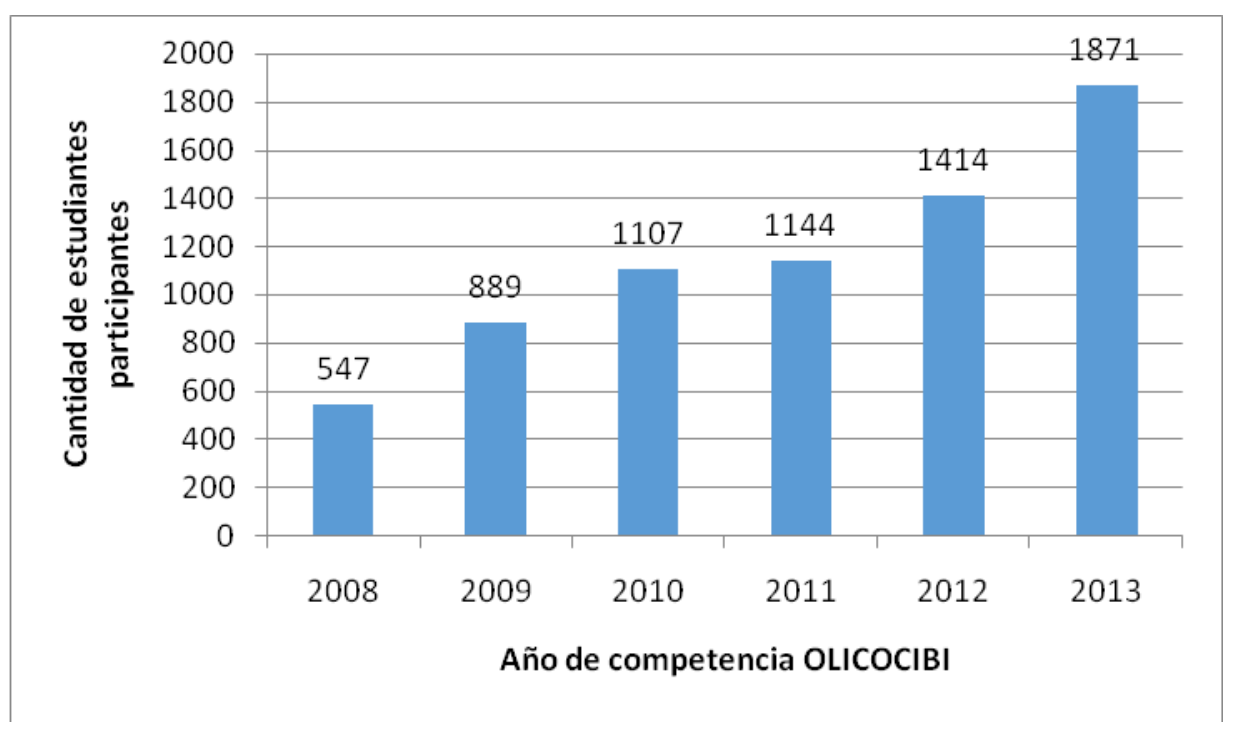

Fuente: Elaboración propia

Figura 2. Cantidad de colegios que han participado por año

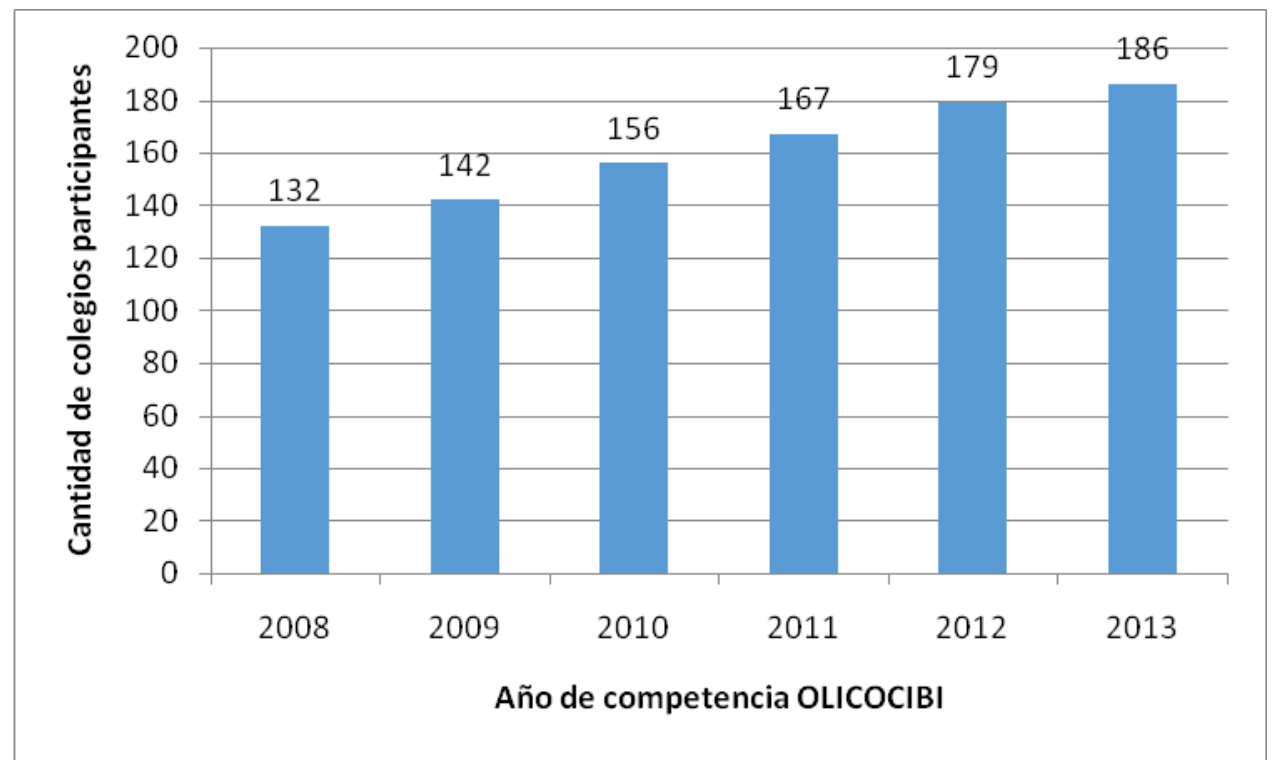

Fuente: Elaboración propia 
Se puede visualizar que para la III competencia, en el año 2009, los colegios que participaron fueron más de 140, pertenecientes a distintas modalidades y regiones del país, con lo que se duplicó la cantidad de estudiantes con respecto al año anterior. Para el 2010 se sumaronla UCR y la UNED, logrando aumentar la participación de los colegios a nivel nacional para un total de 156 centros educativos. Mientras en el 2011 las OLICOCIBI se engalanaron con más de 1100 participantes de 167 colegios, con la colaboración del Colegio de Biólogos de Costa Rica y del Colegio de Licenciados y Profesores en Letras, Filosofía, Ciencias y Artes. Las Olimpiadas de 2012 iniciaron con más de 1400 estudiantes de 179 instituciones,y, finalmente, en el 2013 se alcanzó la cifra record de inscripción de 186 colegios (Pereira, 2013).

Organizar un evento como las OLICOCIBI genera espacios competitivos a nivel educativo, tanto para los y las estudiantes como para el personal docente de secundaria, así como una actualización en los procesos de la enseñanza de las ciencias biológicas, favoreciendo el aprendizaje cognitivo y significativo para llevar a cabo la aplicación de conocimientos científicos y tecnológicos.

\section{Apoyo institucional y disponibilidad de personal para ejecutar el proyecto}

Al ser las OLICOCIBI un proyecto enfocado en la extensión y que se realiza a nivel nacional tomando en cuenta todos los colegios tanto estatales como privados de las veintiún regionales del MEP, se requiere contar con un amplio y variado personal para lograr cumplir con la parte administrativa y con la académica.

Para realizar toda la parte administrativa se direccionan diferentes aspectos a ejecutar en cada universidad, siendo estos dirigidos por cada coordinador 0 coordinadora institucional, que delega algunas actividades a estudiantes con horas beca como asistentes de programas relacionados con la Enseñanza de las 
Ciencias y la Biología; mientras que para cumplir con las gestiones académicas se cuenta con personal académico de las tres universidades, específicamente de la Escuela de Ciencias Biológicas (UNA), la Escuela de Ciencias Exactas y Naturales (UNED) y Escuela de Biología (UCR).

Según Pereira (2012), para cumplir con la parte administrativa y con la académica se deben tomar en cuenta las siguientes gestiones a realizar:

Durante febrero y parte de marzo se hace la divulgación y la inscripción de los (as) estudiantes participantes.

En mayo, se aplica a nivel nacional, en las 12 sedes regionales, un examen teórico para ambas categorías, que consta de 80 preguntas de selección única.

Durante junio, aproximadamente el $20 \%$ de la población estudiantil que aprueba exitosamente la fase teórica eliminatoria o regional, es seleccionado para continuar a la prueba final o nacional. En esta etapa, los competidores de categoría $B$, sólo realizan una prueba teórica; en el caso de quienes participan en la categoría $A$, desarrollan dos pruebas: una teórica y otra experimental (de un valor de 70 y $30 \%$ respectivamente).

Las pruebas regionales y nacionales de ambas categorías, son elaboradas y validadas por académicos (as) de las universidades participantes, sin embargo las actividades logísticas son organizadas por la UNA y apoyadas por la UNED y UCR, con el auspicio del MICIT (p.16).

La parte experimental de la prueba final de la categoría $A$ se desarrolla combinando habilidades y destrezas de laboratorio y de campo; para ello se diseña una práctica que nivela los conocimientos teóricos y prácticos.

A todos los estudiantes con medallas de categoría $A$, conjuntamente con los tres primeros lugares de la categoría B, se les invita a participar en el Programa de 
Preparación para la Participación en las Olimpiadas Iberoamericanas de Biología (OIAB) y la Olimpiada Internacional de Biología (OIB).

Este proceso consiste en fases de preparación individual para cada estudiante, basándose en guías teóricas, experimentales, cuestionarios, material audiovisual y prácticas de laboratorio, material preparado por el comité organizador que busca optimizar el nivel cognitivo, las destrezas y las habilidades de los y las participantes.

Precisamente, cada una de estas acciones ha tenido los resultados esperados, por lo que las OLICOCIBI han logrado integrar la docencia, la investigación y la extensión. Es por ello que las instituciones involucradas en este proyecto lo consideran de gran beneficio, y la Universidad Nacional lo declaró en el 2008 como un evento de interés institucional y, posteriormente, en el 2011 el Ministerio de Educación Pública determinó su importancia en el ámbito educativo nacional, porque amplía la cultura biológica de los y las jóvenes e impulsa las vocaciones científicas, extendiendo las habilidades, las actitudes y los valores del pensamiento racional y científico. Por último, también en el 2011 la Universidad Estatal a Distancia declaró las Olimpiadas como de interés institucional.

Sin embargo, las OLICOCIBI cuentan con el invaluable apoyo de otras unidades académicas, de entes gubernamentales como el MEP, el MICIT yel CONICIT, del Colegio de Licenciados y Profesores en Letras, Filosofía, Ciencias y Artes, del Colegio de Biólogos y de algunas empresas privadas.

Cada año esta actividad logra exitosamente los propósitos formulados y, a pesar de las dificultades que se dan en el proceso, se proyecta su mejoría e innovación en cada periodo. 


\section{Aportes que brindan las OLICOCIBI a la calidad educativa secundaria}

La comisión organizadora que constituye este proyecto - representada por las tres universidades (UNA, UNED y UCR) - brinda a la educación media en el área de las ciencias biológicas el establecimiento de un programa de capacitaciones dirigidas a docentes en zonas rurales y urbanas, la regionalización en doce zonas del país para la aplicación de la prueba eliminatoria y el apoyo al y a la estudiante por medio de capacitaciones y de la participación en las competiciones internacionales (Olimpiada Iberoamericana y Olimpiada Internacional de Biología), lo que involucra un arduo proceso de preparación.

Las universidades discuten en conjunto una serie de actividades prácticas que deben realizar los y las participantes en las diferentes áreas de la biología, así como tipos de capacitaciones de aprendizaje que debe ejecutar el estudiantado que representa al país en las olimpiadas tanto iberoamericanas como internacionales. Estas acciones a desarrollar normalmente son realizadas en la UNA o en la UCR, ya que cuentan con equipo y personal académico especializado. De esta manera, los y las estudiantes adquieren fortalezas en sus procesos de aprendizaje individual y grupal, aumentando sus conocimientos teóricos y prácticos para conseguir una mejor calidad y nivel educativo en el área de las ciencias.

Según Camacho (2011), las OLICOCIBI desempeñan una gran labor en cuanto a la alfabetización científica, ya que logran en la dimensión conceptual, procedimental y actitudinal de los y las participantes el fortalecimientode los procesos de enseñanza-aprendizaje de la Biología, procurando un aprendizaje significativo y promoviendo distintas estrategias que permitan la adquisición de habilidades teóricas, prácticas y procedimentales. 
Se puede manifestar que este tipo de competencias logra en los y las estudiantes una socialización de conocimientos, destrezas, habilidades y actitudes y la producción de pensamiento analítico, crítico y científico de la contextualización actual, para innovar en la adquisición de la enseñanza y del aprendizaje, y por ende tener un mejor nivel cognitivo en la disciplina científica.

Al ser un proyecto en el que participan estudiantes y docentes de diferentes instituciones, se requiere la apertura de espacios donde se promuevan valores importantes, como la solidaridad, la equidad, la responsabilidad, el trabajo en equipo, la competencia sana y el respeto por la naturaleza.

Sin embargo, las universidades también le brindan capacitaciones o talleres al personal docente de educación secundaria que desea una actualización de conocimientos, donde se le refuerza en los diferentes procesos de enseñanza y aprendizaje, así como en la utilización de diferentes métodos y estrategias para abarcar los contenidos en el área de las ciencias biológicas, alcanzando con esto una mejor calidad educativa a nivel secundario.

Por otro lado, las OLICOCIBI tratan de que los talleres o las capacitaciones que se le brindan al profesorado sean orientados a desarrollar destrezas y creatividad en la mediación pedagógica que aplican a sus estudiantes; un aspecto que favorece la búsqueda en el mejoramiento del proceso educativo, porque se requiere que el propio docente realice pequeñas evaluaciones en el centro educativo para lograr identificar a los y las estudiantes con habilidades y actitudes en las ciencias biológicas que puedan participar en estas competencias (Pereira, Camacho y Muñoz, 2013). 
Por lo tanto, a los y las docentes también se les brinda la oportunidad de compartir, socializar y discutir cómo incorporar nuevas estrategias metodológicas en su labor profesional para promover un progreso institucional. De ahí que los docentes se consideren los principales actores en el adecuado proceso de interacción entre la adquisición de conocimientos y el o la estudiante.

De acuerdo a lo comentado anteriormente, los autores Soler, Fleota, Carenton, Cuello, Pérez y Palacios (2006) señalaron que:

El papel fundamental del docente es gestionar, guiar y enseñar contenidos y procedimientos en el aula porque es quien más cerca se encuentra de la formación de los estudiantes. Por lo tanto, es responsable de desarrollar diariamente estrategias pedagógicas que les permitan enfrentar la diversidad de alumnos (as) y entre ellos, diferenciar aquéllos que tienen afinidad y están motivados en aprender más sobre un contenido específico (p.32).

Se puede indicar que el o la docente es uno de los principales inspiradores para motivar alestudiantado, lo que implica para ellos realizar diferentes metodologías que ayuden a los y las estudiantes a asimilar su proceso de enseñanzaaprendizaje. Por eso es necesario que se capacite continuamente a los educadores y las educadoras: para adquirir un mejor proceso pedagógico. Es por ello que sobresale lo expuesto por los autores Berzal y Ortiz (2006).

Es necesario la participación de profesores que tengan buenas ideas como una acción renovadora orientada hacia la mejora educativa, y esto únicamente se llevará a cabo con la activa participación de docentes, que sean capaces de comprender y que deseen conducir cambios reales en las aulas e instituciones. Por ello se ha creído que el docente es el principal motor de inspiración y sobre todo de 
motivación para sus estudiantes, y de él depende que se potencie o no al aprendiente (p.8).

Las Olimpiadas de Biología pretenden que la participación de la persona docente en este tipo de competencias responda a un perfil, donde su función no solo sea la de transmitir conocimientos, sino la de orientar a los y las estudiantes hacia el aprendizaje. Esto hace necesario que la población docente reciba una formación continuaen la que se le brinden capacitaciones, y así ser estimulada permanentemente. Lo anterior facilita el desarrollo de diferentes métodos o técnicas de enseñanza, para así adquirir la capacidad de trabajar en grupo, ser entusiasta, investigativo, participativo, reflexivo, gozar de una excelente comunicación, motivación y actitud positiva, y sobre todo ser responsable de sus compromisos didácticos (Rodríguez, 2012).

Con respecto a las capacitaciones o los talleres que le brinda el comité organizador de las OLICOCIBI a la población docente de secundaria, estos tienen como propósito promover diferentes métodos pedagógicos, así como reforzar, refrescar y aumentar los conocimientos en los temas que hay que desarrollar en los colegios; también dar a conocer temas de interés actual en el área de las ciencias biológicas, porque al estar la ciencia vinculada con la tecnología es probable descubrir algunos cambios que se den a corto plazo.

En la tabla 1 se presentan algunos de los talleres que se les han brindado a docentes de secundaria. Se indica tanto la cantidad de docentes que participaron como la zona donde fue efectuado el taller. Entre los objetivos se encontraban la actualización y la profundización de algunos contenidos temáticos como procesos de enseñanza-aprendizaje que se pueden aplicar en la secundaria, con el propósito de mejorar la calidad educativa. No se ejemplifica la capacitación de los y las estudiantes porque esta depende de los contenidos biológicos que se 
establezcan para cada competencia de las Olimpiadas Iberoamericanas, ya que estos siempre cambian; mientras que para la Olimpiada Internacional se ejecutan todos los temas relacionados con la biología tomando en cuenta tanto la parte teórica como la práctica, y dependerá de la destreza y habilidad de cada estudiante para enfatizar más en algunas áreas.

Tabla 1. Capacitaciones brindadas a los y las docentes de secundaria en el área de las Ciencias (2008-2013)

\begin{tabular}{|c|c|c|c|}
\hline Año & Capacitación & $\begin{array}{l}\text { Cantidad de } \\
\text { participantes }\end{array}$ & $\begin{array}{l}\text { Zona donde se } \\
\text { efectuó la } \\
\text { capacitación }\end{array}$ \\
\hline 2008 & $\begin{array}{l}\text { Importancia de la Biología: } \\
\text { Ciencia, Tecnología y Sociedad }\end{array}$ & 32 & Heredia, UNA \\
\hline 2008 & $\begin{array}{l}\text { Enseñanza Interactiva de la } \\
\text { Biología y Biología Celular y } \\
\text { Aplicaciones }\end{array}$ & 28 & Heredia, UNA \\
\hline 2009 & $\begin{array}{l}\text { Alfabetizando Científicamente a } \\
\text { través de las OLICOCIBI }\end{array}$ & 25 & Heredia, UNA \\
\hline 2009 & $\begin{array}{l}\text { Evolución de Ecosistemas } \\
\text { Marinos y Costeros }\end{array}$ & 24 & $\begin{array}{l}\text { ECMAR, } \\
\text { Puntarenas }\end{array}$ \\
\hline 2009 & $\begin{array}{l}\text { Actualización en el Mundo } \\
\text { Vegetal }\end{array}$ & 30 & Heredia, UNA \\
\hline 2010 & Actualización en Ecología & 36 & $\begin{array}{l}\text { Estación } \\
\text { Experimental } 28 \\
\text { Millas, Guápiles }\end{array}$ \\
\hline 2010 & $\begin{array}{l}\text { Botánica General y sus } \\
\text { Implicaciones en el Manejo y } \\
\text { Conservación de los Recursos } \\
\text { Vegetales }\end{array}$ & 72 & $\begin{array}{l}\text { Nicoya, Liberia y } \\
\text { Cañas, } \\
\text { Guanacaste }\end{array}$ \\
\hline 2010 & $\begin{array}{l}\text { Actualización y Capacitación en } \\
\text { Ecosistemas Vegetales Costeros }\end{array}$ & 23 & $\begin{array}{l}\text { ECMAR, } \\
\text { Puntarenas }\end{array}$ \\
\hline 2010 & \begin{tabular}{lrr} 
Actualización y & Capacitación en \\
Técnicas & \multicolumn{2}{c}{ Moleculares } \\
Novedosas & Aplicadas al \\
Mejoramiento & Genético de \\
Plantas & &
\end{tabular} & 23 & $\begin{array}{l}\text { ECMAR, } \\
\text { Puntarenas }\end{array}$ \\
\hline 2010 & $\begin{array}{l}\text { Abordaje de las Temáticas de } \\
\text { las Estructuras Vegetales para } \\
\text { Profesores de Ciencias }\end{array}$ & 32 & Heredia, UNA \\
\hline
\end{tabular}




\begin{tabular}{|c|c|c|c|}
\hline & Naturales & & \\
\hline 2011 & $\begin{array}{l}\text { Botánica General y sus } \\
\text { Aplicaciones al Desarrollo de } \\
\text { Clases de Ciencias para } \\
\text { Profesores del III Ciclo y la } \\
\text { Educación Diversificada }\end{array}$ & 32 & $\begin{array}{l}\text { Estación } \\
\text { Experimental } 28 \\
\text { Millas, Guápiles }\end{array}$ \\
\hline 2011 & $\begin{array}{l}\text { Botánica General y sus } \\
\text { Aplicaciones al Desarrollo de } \\
\text { Clases de Ciencias para } \\
\text { Profesores del III Ciclo y la } \\
\text { Educación Diversificada }\end{array}$ & 33 & UCR, Limón \\
\hline 2011 & $\begin{array}{l}\text { Actualización en Técnicas de } \\
\text { Campo y Laboratorio Aplicables } \\
\text { en el Desarrollo de Programas } \\
\text { de Alfabetización Científica en } \\
\text { Costa Rica }\end{array}$ & 20 & $\begin{array}{c}\text { ECMAR, } \\
\text { Puntarenas }\end{array}$ \\
\hline 2011 & $\begin{array}{l}\text { Dinámica y Preparación de los } \\
\text { Estudiantes de Secundaria para } \\
\text { la Aplicación de } \\
\text { Nacionales de } \\
\text { Biológicas }\end{array}$ & 20 & $\begin{array}{c}\text { ECMAR, } \\
\text { Puntarenas }\end{array}$ \\
\hline 2011 & $\begin{array}{l}\text { Actualización en la Temática de } \\
\text { Estructura y Funcionamiento de } \\
\text { los Tejidos y Órganos Animales } \\
\text { para Docentes de Ciencias } \\
\text { Naturales de III Ciclo y } \\
\text { Diversificada }\end{array}$ & 27 & Heredia, UNA \\
\hline 2012 & \begin{tabular}{lll} 
Actualización & \multicolumn{2}{c}{ Teórico-Práctica } \\
en Biología & Celular y sus \\
Aplicaciones & &
\end{tabular} & 45 & Heredia, UNA \\
\hline 2013 & $\begin{array}{l}\text { Actualización Teórico-Práctica } \\
\text { en Ecología General }\end{array}$ & 35 & Heredia, UNA \\
\hline
\end{tabular}

Fuente: Pereira (2013). Presentación del informe final a la comisión organizadora de las Olimpiadas Costarricenses de Ciencias Biológicas.

La finalidad de este espacio competitivo es promover el desarrollo de la educación científica, estimular la participación de estudiantes y docentes en la mejora de sus conocimientos y lograr la adquisición de nuevas destrezas y habilidades 
relacionadas con las ciencias biológicas, campo en el que se pretende mejorar la educación secundaria.

\section{Logros obtenidos en las Olimpiadas Iberoamericanas}

Costa Rica inició las Olimpiadas a nivel nacional en el 2007, y para el 2008 inició su participación en las competencias cognitivas de las Olimpiadas Iberoamericanas de Biología (OIAB), las cuales se llevaron a cabo la primera vez en el año 2007 en la Ciudad de México (Escuela de Ciencias Biológicas, 2008).

En el año 2008 se compitió a nivel lberoamericano y el país organizador fue Brasil. En estas Olimpiadas se contó con una delegación costarricense conformada por un académico y una académica del comité organizador del proyecto y dos estudiantes ganadores de la I Olimpiada Costarricense de Ciencias Biológicas; en esta primera participación se obtuvo una medalla de bronce. Sin duda, fue una experiencia constructiva y enriquecedora en el área de la biología para Costa Rica, donde se compartió social, cultural, educativa y científicamente con delegaciones de países latinos (Olimpiadas Costarricenses de Ciencias Biológicas, 2008).

Las competencias a nivel Iberoamericano han sido una gran experiencia tanto para la comisión organizadora de Costa Rica como para los y las estudiantes que han participado en estas justas, ya que durante la preparación de estos eventos internacionales lo que se requiere es potenciar las destrezas cognitivas de la biología, apropiándose de los conceptos, así como su aplicación en la vivencialidad y práctica cuando se enfrenta al uso de los diversos equipos de laboratorio, integrando el abordaje teórico y el práctico; sin embargo, para lograr este proceso es indispensable la actitud del o de la estudiante, de ahí la importancia de abarcar aspectos motivacionales, culturales y valorativos en los 
que influyan el respeto, la dedicación, la responsabilidad, el compromiso y la confianza en sí mismo.

De acuerdo con lo expuesto anteriormente, se menciona la experiencia vivida por Daniel Chacón, quien fue uno de los participantes que representó a Costa Rica en las Olimpiadas Iberoamericanas de Biología de 2010, donde manifestó que las Olimpiadas Iberoamericanas le dejaron una gran experiencia enriquecedora en varios aspectos, como el académico, el social, el cívico, el personal y el vocacional.

Académicamente, las OLICOCIBI logran desarrollar habilidades intelectuales y manuales requeridas por el científico o la científica para obtener un aprendizaje que forje las bases académicas que permitirán un desenvolvimiento pleno en la Carrera de Ciencias Biológicas, y de esta manera llegar a ser personas multifacéticas capaces de entender, apreciar y participar en todas las áreas de la biología, ya que los conocimientos son adquiridos para toda la vida. En el área vocacional se adquiere acercamiento a la realidad profesional de las carreras científicas, como el descubrimiento y/o la reformación de la vocación científicobiológica, para apreciar el trabajo de la comunidad científica y, por último, aprender a amar lo que se hace.

En la parte social se aprende a trabajar en equipo, con comunicación asertiva, compañerismo y solidaridad, además se forjan amistades duraderas y muy apreciadas que incluso traspasan las fronteras; las OLICOCIBI se visualizan como una gran familia.

Con respecto a lo personal, se adquiere disciplina, compromiso, responsabilidad, diligencia, respeto, una mejor administración del tiempo y una mentalidad positiva 
sin autolimitarse para trabajar por lo que se quiere. De esta manera, se aprende a ser un mejor estudiante hasta alcanzar la superación personal y la satisfacción por una tarea bien finalizada (Comisión Organizadora de las Olimpiadas Costarricenses de Ciencias Biológicas, 2013).

La experiencia, desde el punto de vista de la organización, ante un evento a nivel internacional se fue adquiriendo desde el primer año de participación (2008), y es por ello que para el 2011 se logró que Costa Rica fuera la sede para realizar las Olimpiadas Iberoamericanas. Debido a que se trabajó arduamente y con mucho esfuerzo desde meses antes de la ejecución se obtuvieron grandes éxitos a nivel organizativo y a nivel académico, incluso en esta Olimpiada se realizó por primera vez la participación de los y las estudiantes en un evento denominado Rally, en el cual la actividad era totalmente recreativa, con el propósito de conseguir una mejor unión y convivencia cultural entre las personas participantes de todos los países competidores.

Para las justas de 2011 se obtuvieron a nivel académico dos medallas de oro y una de plata, afirmando Costa Rica su gran avance a nivel competitivo en las Olimpiadas Iberoamericanas.

Seguidamente se presentan en la tabla 2 los años en los que se ha competido a nivel Iberoamericano, así como los y las estudiantes participantes y las medallas obtenidas en cada Olimpiada. 
Tabla 2. Medallas obtenidas por los y las estudiantes participantes en las Olimpiadas Iberoamericanas (2008-2013)

\begin{tabular}{|c|c|c|c|c|}
\hline Año & Evento & Sitio & Delegación & Logro \\
\hline 2008 & II OIAB & Brasil & $\begin{array}{l}\text { Kenneth Stelle (competidor) } \\
\text { Oscar Umaña (competidor) }\end{array}$ & 1 medalla de bronce \\
\hline 2009 & $\begin{array}{l}\text { III } \\
\text { OIAB }\end{array}$ & España & $\begin{array}{l}\text { Dagoberto Herrera } \\
\text { (competidor) } \\
\text { Stefani Díaz (competidora) } \\
\text { Diana Rojas (competidora) } \\
\text { LitzyXu (competidora) }\end{array}$ & 1 medalla de bronce \\
\hline 2010 & $\begin{array}{l}\text { IV } \\
\text { OIAB }\end{array}$ & Perú & $\begin{array}{l}\text { Daniel Chacón (competidor) } \\
\text { Richard Mejías (competidor) } \\
\text { Marco Castro (competidor) }\end{array}$ & $\begin{array}{l}2 \text { medallas de oro y } 1 \\
\text { medalla de plata }\end{array}$ \\
\hline 2011 & V OIAB & $\begin{array}{l}\text { Costa } \\
\text { Rica }\end{array}$ & $\begin{array}{l}\text { Luis Carlos Montiel } \\
\text { (competidor) } \\
\text { Mario Méndez (competidor) } \\
\text { Juan Cambronero } \\
\text { (competidor) } \\
\text { Alexa Jiménez (competidora) }\end{array}$ & $\begin{array}{l}2 \text { medallas de oro y } 1 \\
\text { medalla de plata }\end{array}$ \\
\hline 2012 & $\begin{array}{c}\mathrm{VI} \\
\text { OIAB }\end{array}$ & Portugal & $\begin{array}{l}\text { Ariel Meléndez Resenterra } \\
\text { (competidora) } \\
\text { Rodson Corrales Muñoz } \\
\text { (competidor) } \\
\text { Erick Rojas Hidalgo } \\
\text { (competidor) }\end{array}$ & $\begin{array}{l}2 \text { medallas de oro y } 1 \\
\text { medalla de plata }\end{array}$ \\
\hline 2013 & $\begin{array}{l}\mathrm{VI} \\
\text { OIAB }\end{array}$ & Argentina & $\begin{array}{l}\text { Sebastián Hernández } \\
\text { Cifuentes (competidor) } \\
\text { Álvaro Vega Hidalgo } \\
\text { (competidor) } \\
\text { Esteban Barquero Fernández } \\
\text { (competidor) }\end{array}$ & $\begin{array}{l}2 \text { medallas de plata y } \\
1 \text { medalla de bronce }\end{array}$ \\
\hline 2014 & $\begin{array}{l}\text { VIII } \\
\text { OIAB }\end{array}$ & México & Melissa Salazar(competidor) & 1 medalla de bronce. \\
\hline
\end{tabular}

Fuente: Elaboración propia basada en los registros de participación 
Cabe destacar que la delegación de Costa Rica participó por primera vez en el 2013 con dos estudiantes en las Olimpiadas Internacionales realizadas en Suiza, en las cuales se compitió con los mejores estudiantes de Biología a nivel mundial, obteniendo uno de los estudiantes una mención honorífica muy importante para nuestro país. Gracias a la participación de estos dos estudiantes Costa Rica logró ser reconocida en la disciplina de las ciencias biológicas como un país competitivo en las mayores justas de biología. Los estudiantes adquirieron un gran intercambio de experiencias culturales y académicas para llegar a ser futuros profesionales con un amplio potencial en pensamiento analítico, critico, racional y científico.

\section{Carreras profesionales de preferencia que escogióla población estudiantil exolímpica que representó a Costa Rica en las Olimpiadas Iberoamericanas (OIAB)}

Como valor agregado, las OLICOCIBI son una actividad alfabetizadora en las ciencias biológicas; permiten identificar estudiantes con potencial y facultades meritorias, tanto en el estudio de las ciencias biológicas y sus múltiples ramas como en la creatividad, la perseverancia y la capacidad analítica (Escuela de Ciencias Biológicas, 2009).

Para Furió, Vilches, Guisasola y Romo (2001), citados por Camacho (2011), la alfabetización científica está orientada a la enseñanza científica vinculada a aspectos sociales y personales de los y las estudiantes. La ciencia es parte esencial de la formación básica ciudadana, por lo que no puede alejarse de los currículos escolares, sino que tiene que promover en la mayoría de la población la disposición de conocimientos científicos precisos para desenvolverse diariamente, que le ayuden a resolver problemas de salud y supervivencia básica y para reflexionar sobre la ciencia como parte de la cultura, y de esta manera seguir aprendiendo de ella después de la escolarización formal. 
Tomando en cuenta la información anterior, se puede determinar que si a un estudiante de los niveles escolar y colegial se le inculca la adquisición de conocimientos en el área de las ciencias biológicas, este dispondrá de alfabetización en la enseñanza de las Ciencias como parte de su vida social y personal de manera natural, y si esta actividad le proporciona interés, motivación, compromiso, entre otros aspectos, le ayudará a ser un futuro profesional en el ámbito científico.

Costa Rica, por medio del MEP, el MICIT y el CONICIT, promueve e impulsa en los estudiantes propuestas de motivación, una actitud investigativa, compromiso y responsabilidad ante los avances científicos y tecnológicos en el campo de la biología. Por lo tanto, las OLICOCIBI tratan de consolidar este tipo de propuestas con las actividades que se ejecutan dentro de este proyecto para fortalecer aún más el área científico-tecnológica que muchade la población estudiantil trae innatamente y que termina de desarrollarla cuando participa en este tipo de competencias, dejando como resultado que la mayoría de los y las estudiantes participantes que hoy en día son exolímpicos se inclinaron a escoger carreras profesionales vinculadas con el área de las ciencias.

En la tabla 3 se presentan las personas participantes ex olímpicas de la OIAB desde el año 2008 hasta el 2013 y las carreras profesionales que han escogido en las ramas de la biología.

Tabla 3. Ex olímpicos de la OIAB desde el año 2008 hasta el 2013 y carreras de preferencia

\begin{tabular}{|c|l|l|}
\hline Nombre & $\begin{array}{c}\text { Centro educativo de estudios } \\
\text { secundarios }\end{array}$ & \multicolumn{1}{c|}{$\begin{array}{c}\text { Carreras que está } \\
\text { cursando }\end{array}$} \\
\hline Kenneth Stelle & Liceo de Costa Rica & Medicina, UCR \\
\hline
\end{tabular}


Unión interuniversitaria para el fortalecimiento de las Olimpiadas Costarricenses de Ciencias Biológicas como proceso de formación de los futuros profesionales en el área de las ciencias y la actualización de los conocimientos de los y las docentes de secundaria sobre biología

Magaly Rodríguez Calvo y José M. Pereira Chaves

\begin{tabular}{|c|c|c|}
\hline Oscar Umaña* & $\begin{array}{l}\text { Colegio Científico } \\
\text { Costarricense, Sede Alajuela }\end{array}$ & Medicina, UCR \\
\hline $\begin{array}{l}\text { Dagoberto } \\
\text { Herrera* }\end{array}$ & $\begin{array}{l}\text { Colegio Científico } \\
\text { Costarricense, Sede Alajuela }\end{array}$ & $\begin{array}{l}\text { Ciencias Económicas, } \\
\text { Universidad Autónoma de } \\
\text { Monterrey, México }\end{array}$ \\
\hline Stefani Díaz* & $\begin{array}{l}\text { Colegio Humanístico } \\
\text { Costarricense, Sede Heredia }\end{array}$ & $\begin{array}{l}\text { Biología, UNA y Periodismo, } \\
\text { UCR }\end{array}$ \\
\hline Diana Rojas* & $\begin{array}{l}\text { Colegio Humanístico } \\
\text { Costarricense, Sede Heredia }\end{array}$ & $\begin{array}{l}\text { Relaciones Internacionales, } \\
\text { Universidad de Harding, } \\
\text { Estados Unidos }\end{array}$ \\
\hline LitzyXu & $\begin{array}{l}\text { Colegio Científico Bilingüe } \\
\text { Reina de los Ángeles }\end{array}$ & Ingeniería Química, UCR \\
\hline Daniel Chacón* & $\begin{array}{l}\text { Colegio Científico } \\
\text { Costarricense, Sede San Pedro }\end{array}$ & $\begin{array}{l}\text { Microbiología, UCR y } \\
\text { Biología, UNA }\end{array}$ \\
\hline Richard Mejías* & $\begin{array}{l}\text { Colegio Científico } \\
\text { Costarricense, Sede San } \\
\text { Ramón }\end{array}$ & $\begin{array}{l}\text { Ingeniería Química, UCR y } \\
\text { Biología, UNA }\end{array}$ \\
\hline Marco Castro* & $\begin{array}{l}\text { Colegio Científico } \\
\text { Costarricense, Sede Cartago }\end{array}$ & $\begin{array}{l}\text { Veterinaria, UNA y Biología, } \\
\text { UCR }\end{array}$ \\
\hline $\begin{array}{l}\text { Luis Carlos } \\
\text { Montiel* }^{*}\end{array}$ & $\begin{array}{l}\text { Colegio Científico } \\
\text { Costarricense, Sede Liberia }\end{array}$ & Biología, UCR \\
\hline Mario Méndez* & $\begin{array}{l}\text { Colegio Humanístico } \\
\text { Costarricense, Sede Heredia }\end{array}$ & $\begin{array}{l}\text { Ingeniería Aeroespacial en } \\
\text { Samara State Aerospace } \\
\text { University, Russia }\end{array}$ \\
\hline $\begin{array}{l}\text { Juan } \\
\text { Cambronero* }\end{array}$ & $\begin{array}{l}\text { Colegio Científico } \\
\text { Costarricense, Sede San Pedro }\end{array}$ & $\begin{array}{l}\text { Microbiología y Biología, } \\
\text { UCR }\end{array}$ \\
\hline Alexa Jiménez & $\begin{array}{l}\text { Colegio Científico Bilingüe } \\
\text { Reina de los Ángeles }\end{array}$ & Física y Biología, UCR \\
\hline Ariel Meléndez & $\begin{array}{l}\text { Colegio Científico de San } \\
\text { Ramón }\end{array}$ & Medicina Veterinaria, UNA \\
\hline Rodson Corrales & $\begin{array}{l}\text { Colegio Científico Bilingüe } \\
\text { Reina de los Ángeles }\end{array}$ & Ingeniería Química, UCR \\
\hline Erick Rojas & Colegio los Ángeles & Microbiología, UCR \\
\hline
\end{tabular}


Unión interuniversitaria para el fortalecimiento de las Olimpiadas Costarricenses de Ciencias Biológicas como proceso de formación de los futuros profesionales en el área de las ciencias y la actualización de los conocimientos de los y las docentes de secundaria sobre biología

Magaly Rodríguez Calvo y José M. Pereira Chaves

\begin{tabular}{|l|l|l|}
\hline $\begin{array}{l}\text { Sebastián } \\
\text { Hernández }\end{array}$ & $\begin{array}{l}\text { Colegio Científico } \\
\text { Costarricense, Sede San Pedro }\end{array}$ & Ingeniería Química, UCR \\
\hline Álvaro Vega & $\begin{array}{l}\text { Colegio Humanístico } \\
\text { Costarricense, Sede Heredia }\end{array}$ & Biología, UNA \\
\hline $\begin{array}{l}\text { Esteban } \\
\text { Barquero }\end{array}$ & $\begin{array}{l}\text { Colegio Científico Bilingüe } \\
\text { Reina de los Ángeles }\end{array}$ & Biología, UCR \\
\hline
\end{tabular}

Fuente: Pereira (2014). Informe parcial del proyecto Olimpiadas Costarricenses de Ciencias Biológicas.

*Realizan sus estudios con apoyo económico de becas otorgadas en sus universidades, dado que tienen condiciones económicas limitantes.

\section{Conclusiones}

Las Olimpiadas Costarricenses de Ciencias Biológicas son un espacio competitivo que promueve el desarrollo de la enseñanza y del aprendizaje de las Ciencias Biológicas y estimula la participación de estudiantes y docentes en la mejora de sus conocimientos y en la adquisición de nuevas destrezas y habilidades relacionadas con el ámbito de estas ciencias.

Esta actividad cognitiva alcanzó exitosamente los propósitos formulados con la integración de tres de las universidades más importantes del país pertenecientes a CONARE, lo que ha generado el trabajo armonioso e iniciativas conjuntas de compromiso.

Al ser la persona docente la principal gestoray guía que brinda conocimientos a los y las estudiantes, las OLICOCIBI, por medio de sus capacitaciones y talleres, logran reforzar, refrescar y aumentar conocimientos relacionados con el área de las ciencias.También brindan espacios para compartir, trabajar en equipo, socializar, discutir y sobre todo incorporar nuevas estrategias metodológicas que se pueden aplicar en la enseñanza y el aprendizaje de la Biología. 
En las actividades promovidas y ejecutadas como parte de las OLICOCIBI se ha determinado que las personas participantes han compartido experiencias, como la oportunidad de trabajar en equipo desarrollando prácticas, un análisis de diferentes pruebas y capacitándose en áreas de su interés.

Es evidente el impacto social positivo de este proyecto y del trabajo colaborativo entre las universidades estatales. La viabilidad educativa a nivel nacional se determinó tanto por los altos niveles de interés y participación de la comunidad educativa costarricense como por el interés institucional y de los entes colaboradores medulares en el ámbito gubernamental.

Seguir generando espacios de discusión, análisis y capacitación en las OLICOCIBI se visualiza como una actividad de gran alcance y significado para el desarrollo de las ciencias biológicas en la educación secundaria y en la sociedad costarricense.

La experiencia vivida por los estudiantes ex olímpicos Iberoamericanos ha influido en su escogencia de una carrera profesional, ya que la participación en este evento les proporcionó interés, motivación, una actitud investigativa, compromiso y habilidades intelectuales que funcionan como bases académicas para desenvolverse en las carreras relacionadas con el área de las ciencias y la tecnología, ya que los conocimientos adquiridos son para toda la vida.

En el campo de la biología Costa Rica se mantiene como un fuerte competidor a nivel Iberoamericano, superando a países con una tradición competitiva más amplia. A nivel internacional se evidencia un fuerte potencial que se puede desarrollar con un adecuado programa de preparación para las personas participantes; por ello son necesarios el trabajo y la colaboración en equipo entre las diferentes instituciones educativas a nivel superior y los entes de apoyo. 


\section{Referencias}

Berzal, M. y Ortiz, M. (2006).El papel de las reuniones científicas y educativas en la promoción de la investigación, la innovación y la formación del profesorado de biología. Interaccionar para cambiar y renovar. Revista de Educación en Biología, 9(1), 6-13

Camacho, S. (2011). Aportes del proyecto OLICOCIBI en la alfabetización científica de sus participantes en sus dimensiones conceptual, procedimental y actitudinal (tesis de licenciatura en Enseñanza de las Ciencias). Universidad Nacional, Heredia, Costa Rica.

Comisión Organizadora de las Olimpiadas Costarricenses de Ciencias Biológicas. (Setiembre, 2013). Presentación final de los logros obtenidos en las Olimpiadas de Biología en los años 2010 a 2013 con fondos de CONARE. Primer Jornada de acción social y extensión de CONARE [Power Point]. Consejo Nacional de Rectores de Costa Rica, San José, Costa Rica.

Escuela de Ciencias Biológicas (2008). Proyecto Fortalecimiento y Consolidación de los Programas de Olimpiadas Costarricenses de Ciencias Biológicas e Internacionales de Biología, para mejorar la formación de los y las estudiantes preuniversitarias y los y las estudiantes universitarias con interés y afinidades hacia las Ciencias Biológicas. Universidad Nacional, Heredia, Costa Rica.

Escuela de Ciencias Biológicas (2009). Proyecto Fortalecimiento y Consolidación de los Programas de Olimpiadas Costarricenses de Ciencias Biológicas e Internacionales de Biología, para mejorar la formación de los y las estudiantes preuniversitarias y los y las estudiantes universitarias con interés y afinidades hacia las Ciencias Biológicas. Universidad Nacional, Heredia, Costa Rica.

Olimpiadas Costarricenses de Ciencias Biológicas (2008). Antecedentes. Recuperado de http://www.una.ac.cr/olicocibi/index.php?option=com_content\&task=view\&id $=13 \&$ Itemid $=33$

Pereira, J. (2012). Informe final del proyecto Olimpiadas Costarricenses de Ciencias Biológicas: Primer quinquenio. Universidad Nacional, Heredia, Costa Rica. 
Unión interuniversitaria para el fortalecimiento de las Olimpiadas Costarricenses de Ciencias Biológicas como proceso de formación de los futuros profesionales en el área de las ciencias y la actualización de los conocimientos de los y las docentes de secundaria sobre biología

Magaly Rodríguez Calvo y José M. Pereira Chaves

Pereira, J. (Noviembre, 2013). Presentación del informe final a la comisión organizadora de las Olimpiadas Costarricenses de Ciencias Biológicas [Power Point]. Universidad Nacional, Universidad de Costa Ricay Universidad Estatal a Distancia, Costa Rica.

Pereira, J. (2014). Informe parcial del proyecto Olimpiadas Costarricenses de Ciencias Biológicas: Primer quinquenio. Universidad Nacional, Heredia, Costa Rica.

Pereira, J., Camacho, S. y Muñoz, N. (2013). Las olimpiadas de ciencias biológicas y su papel en la comunidad educativa nacional mediante la integración del competir, compartir, convivir y aprender. Revista Uniciencia, $27(1), 245-265$.

Rodríguez, M. (2012). Olimpiadas Costarricenses de Ciencias Biológicas como proceso fundamental en el mejoramiento de la calidad educativa secundaria de Costa Rica en el área de la biología. Revista Calidad en la Educación Superior, 3(2), 122-140.

Soler, L., Fleota, A., Carenton, J., Cuello, P., Pérez, P. y Palacios, M. (2006). El uso de una especie en extinción como herramienta en Educación Ambiental: experiencias pedagógicas en la Provincia de Corrientes, Argentina. Revista de Educación Biología, 9(2), 29-35. 